\title{
Beta-bloker hedef dozun altında olan olgularda sakubitril/valsartan başlanabilir mi ve başlanırsa klinik yarar sağlar mı?
}

\author{
Dr. Yılmaz Güneş
}

Bolu Abant İzzet Baysal Üniversitesi Tıp Fakültesi, Kardiyoloji Anabilim Dalı, Bolu

Beta-blokerler (BB) düşük ejeksiyon fraksiyonlu kalp yetmezliği (DEFKY) tedavisinin temel taşlarından olup ölüm ve kalp yetmezliği (KY) nedenli hastane yatışlarını azalttıkları ve yaşam kalitesinde düzelme sağladıkları çalışmalarda tutarlı bir şeklilde gösterilmiştir. ${ }^{[1]}$

Bisoprolol, metoprolol ve karvedilol ile NYHA II-IV KY hastalarında yapılan çalışmalarda mortalitede yaklaşık \%35 azalma saptanmıştır. ${ }^{[2]}$ Kılavuzlarda kararlı klinik durumu olan DEFKY hastalarında BB'lerin düşük dozda başlanarak tolere edilebilen maksimum dozlara titre edilmesi tavsiye edilmektedir. Ancak çok sayıda çalışma verisi birikimine ve klinik tecrübeye rağmen klinik pratikte optimum veya tolere edilebilen maksimum BB doza ulaşma konularında sorunlar devam etmektedir.

COPERNICUS (Carvedilol Prospective Randomized Cumulative Survival) çalışmasında anjiyotensin dönüştürücü enzim inhibitörleri (ACEI) [veya anjiyotensin reseptör blokerleri (ARB)] ile diüretik tedavi almakta olan, hacim yüklenmesi olmayan, istirahat ya da minimal eforla semptomatik olan sol ventrikül ejeksiyon fraksiyonu (LVEF) $<\% 25$ olan hastalar plasebo ya da karvedilole randomize edilerek ortalama 10,4 ay takip edildiler. Başlangıçta $2 \times 3.125 \mathrm{mg} / \mathrm{g}$ olan karvedilol dozu 2 hafta aralıklarla sirasiyla $2 \times 6.25,2 \times 12.5$ ve $2 \times 25 \mathrm{mg} / \mathrm{g}$ doza titre edildi. Y1llik mortalite karvedilol ile \%12.8, plasebo ile \%19.7 ve risk azalması \%35 bulundu $(\mathrm{p}=0.00004)$. KY nedenli hastane yatışları karvedilol ile \%40 azald $1(\mathrm{p}<0.0001)$. Doz titrasyonu açısından üç aylık dönemde hastaların \%77.6'sında hedef doza ulaşılabildi ve $\% 70.5$ 'i çalışma süresince bu tedavi dozunu idame edebildi. Takip döneminde hastaların \%38.3'inde doz azaltılmış, 12.6\%'sında ise karvedilol tamamen bırakılmıştı. ${ }^{[3]}$ USCS (U.S Carvedilol HF Study) çalışmasında ise karvedilol 2x6.25 veya $2 \times 12.5 \mathrm{mg} / \mathrm{g}$ dozunda başlandı. Ortalama karvedilol dozu $45 \pm 27 \mathrm{mg}$ olup $2 \times 25 \mathrm{mg}$ hedef dozu hastaların $\% 80$ 'ine verilebildi (Tablo 1). ${ }^{[4]}$

CIBIS II (Cardiac Insufficiency Bisoprolol Study) çalışmasında LVEF <\%35, NYHA III-IV olan DEFKY hastalarında bisoprolol 1x1.25 mg/g den başlanarak $1 \times 10 \mathrm{mg} / \mathrm{g}$ hedef dozuna titre edildi. Ortalama 1.3 y1l takip döneminde bisoprolol tüm nedenli mortaliteyi \%34, KV mortaliteyi \%29, KY nedenli hastane yatışını $\% 36$ azalttı. $10 \mathrm{mg} / \mathrm{g}$ dozuna hastaların sadece 42\%'sinde ulaşılabildi. Hastaların yarısında hedef dozun $>\% 75$ 'i idame edilebildi (Tablo 1 ). ${ }^{[5]}$

MERIT-HF çalışmasında, LVEF <\%40 ve NYHA II-IV hastalara metoprolol süksinat $1 \times 12.5 \mathrm{mg} /$ gün (NYHA III-IV) ya da $1 \times 25 \mathrm{mg} /$ gün (NYHA II) başlanıp 8 haftanın üzerinde hedef doz olan $200 \mathrm{mg}$ 'a çıkıldı. Hastaların \%64'ünde $200 \mathrm{mg} / \mathrm{g}$ hedef doza ulaşılırken $\% 87$ 'sinde $>100 \mathrm{mg} / \mathrm{g}$ doz sağlandı. Ortalama metoporolol dozu günlük 159 mg'dı. Ortalama bir yıllık takipte plaseboya göre tüm nedenli mortalitede \%34, KY'ye bağlı mortalitede \%49 görece risk azalması sağlandı (Tablo 1). ${ }^{[6]}$

DEFKY'de, ACEİ/ARB ve BB'ler sinif I endikasyon ile önerilmektedir. Ayrıca tolere edilebilen maksimum hedef dozlara çıkılması tavsiye edilmektedir. Ancak gerçek yaşamda BB dozları genellikle hedef dozun altında kalmaktadır. Nitekim OPTIMIZE-HF (Organized Program to Initiate Lifesaving Treatment in Hospitalized Patients With Heart Failure) kayıt çalışmasında KY nedeniyle hastaneye yatırılan hastalarda yatış sırasında ortalama BB dozu hedef dozun yarısı kadar olup taburculukta hastaların $<\% 10$ 'u hedef dozu almaktaydı. Taburculuk sonrası hedef dozda BB alanların oranı 60 günde $\% 17.5$ ve 90 günde $\% 7.9$ idi. $^{[7]}$ Yaşlılarda bisoprolol ve karvedilolün tolerabilitesinin değerlendirildiği CIBIS-ELD (Cardiac Insufficiency Bisoprolol Study in Elderly) çalışmasinda da hastaların sadece \%31'inde hedef BB dozuna ulaşılmıştı. ${ }^{[8]}$ 
Tablo 1. Kalp yetmezliğindeki bazı ana çalışmalarda beta-bloker dozları

\begin{tabular}{lcccc}
\hline & USCS $(\mathrm{n}=1094)$ & MERIT-HF $(\mathrm{n}=3991)$ & CIBIS II $(\mathrm{n}=2647)$ & COPERNICUS $(\mathrm{n}=2708)$ \\
\hline Beta-bloker & Karvedilol & Metoprolol süksinat & Bisoprolol & Karvedilol \\
Başlama dozu & $2 \times 6.25 \mathrm{mg} / \mathrm{g}$ & $1 \times 12.5 \mathrm{mg} / \mathrm{g}$ & $1 \times 1.25 \mathrm{mg} / \mathrm{g}$ & $2 \times 3.125 \mathrm{mg} / \mathrm{g}$ \\
Hedef doz & $2 \times 25-50 \mathrm{mg} / \mathrm{g}$ & $1 \times 200 \mathrm{mg} / \mathrm{g}$ & $1 \times 10 \mathrm{mg} / \mathrm{g}$ & $2 \times 50-100 \mathrm{mg} / \mathrm{g}$ \\
Ortalama doz & $45 \mathrm{mg} / \mathrm{g}$ & $159 \mathrm{mg} / \mathrm{g}$ & $7.5 \mathrm{mg} / \mathrm{g}$ & $37 \mathrm{mg} / \mathrm{g}$ \\
Titrasyon süresi & $2-10 \mathrm{hafta}$ & $1-8 \mathrm{hafta}$ & $1-15 \mathrm{hafta}$ & $1-8 \mathrm{hafta}$ \\
Ölümde azalma & $65 \%(\mathrm{p}<0.001)$ & $34 \%(\mathrm{p}<0.001)$ & $34 \%(\mathrm{p}<0.001)$ & $35 \%(\mathrm{p}<0.001)$ \\
\hline
\end{tabular}

USCS: U.S Carvedilol HF Study; CIBIS II: Cardiac Insufficiency Bisoprolol Study.

Tablo 2. PARADIGM-HF çalışmasında bazal beta-bloker dozuna göre klinik sonlanımlar

\begin{tabular}{|c|c|c|c|c|c|c|c|c|}
\hline \multirow[b]{2}{*}{ BB dozu } & \multicolumn{4}{|c|}{ Birincil son nokta } & \multicolumn{4}{|c|}{ Kardiyovasküler ölüm } \\
\hline & $\begin{array}{l}\text { Enalapril } \\
\mathrm{n} / \mathrm{N}(\%)\end{array}$ & $\begin{array}{c}\text { Sacubitril/valsartan } \\
\text { n/N (\%) }\end{array}$ & $\mathrm{HR}(95 \% \mathrm{Cl})$ & $\begin{array}{l}\text { Etkileşim } \\
\text { p değeri }\end{array}$ & $\begin{array}{l}\text { Enalapril } \\
\mathrm{n} / \mathrm{N}(\%)\end{array}$ & $\begin{array}{c}\text { Sacubitril/valsartan } \\
\mathrm{n} / \mathrm{N}(\%)\end{array}$ & $\mathrm{HR}(95 \% \mathrm{Cl})$ & $\begin{array}{l}\text { Etkileşim } \\
\text { p değeri }\end{array}$ \\
\hline$<50 n=4167$ & $\begin{array}{c}566 / 2123 \\
(26.7)\end{array}$ & $\begin{array}{c}455 / 2044 \\
(22.3)\end{array}$ & $\begin{array}{c}0.82 \\
(0.72-0.93)\end{array}$ & 0.973 & $\begin{array}{c}353 / 2123 \\
(16.6)\end{array}$ & $\begin{array}{c}289 / 2044 \\
(14.1)\end{array}$ & $\begin{array}{c}0.85 \\
(0.73-0.99)\end{array}$ & 0.923 \\
\hline$\geq 50 n=3644$ & $\begin{array}{c}440 / 1789 \\
(24.6)\end{array}$ & $\begin{array}{c}390 / 1855 \\
(21.0)\end{array}$ & $\begin{array}{c}0.82 \\
(0.72-0.94)\end{array}$ & & $\begin{array}{c}258 / 1789 \\
(14.4)\end{array}$ & $\begin{array}{c}229 / 1855 \\
(12.4)\end{array}$ & $\begin{array}{c}0.84 \\
(0.70-1.0)\end{array}$ & \\
\hline
\end{tabular}

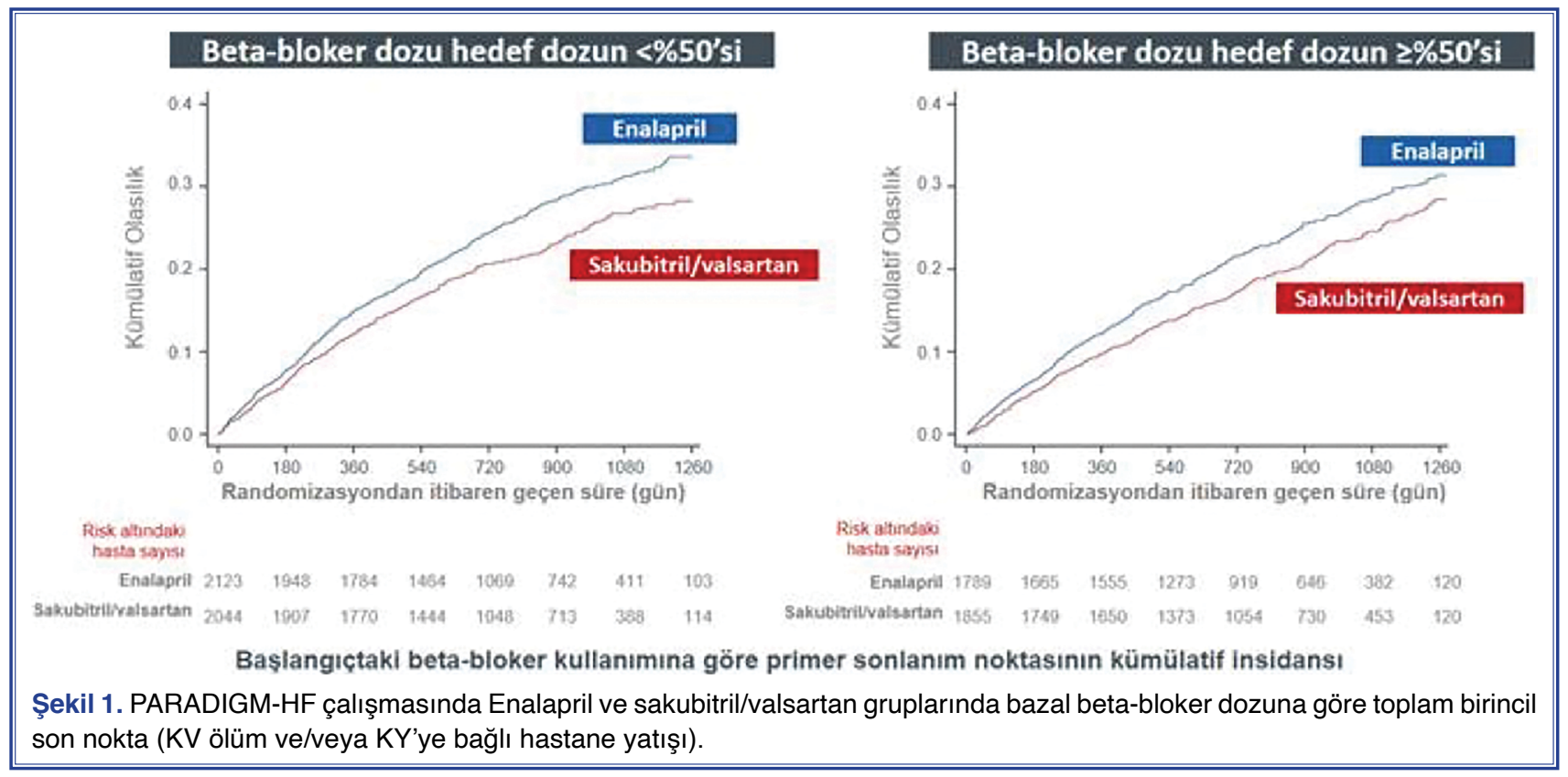

QUALIFY çalışmasına KY hospitalizasyon sonrası $1-15$ ay takip edilen 7092 hasta alındı. Kılavuz önerileri doğrultusunda hastaların ACEİ, ARB, BB, MRA ve ivabradin alma uyumlulukları araştırıldı. Uyum skoru hastaların \%67'sinde iyi, \%25'inde 1lım$11, \% 8$ 'sinde ise kötü bulundu. Hedef doz ve $\geq 50$ hedef doz alma oranları ACEİ için \%27.9 ve \%63.3, ARB için $\% 6.9$ ve $\% 39.5$, ivabradin için $\% 6.9$ ve $\% 39.5$ ve BB için \%14.8 ve \%51.8 idi. ${ }^{[9]}$
Kalp yetmezliğinde BB dozu mu yoksa hedef kalp hızına ulaşmak mı daha önemli sorusunun araştırıldığı bir çalışmada 2007 ile 2015 yılları arası KY nedeniyle düşük ve yüksek doz BB (karvedilol, metoprolol) alan 36174 hasta verisi incelendi ve sağlanan kalp h1zından bağımsız olarak yüksek doz BB alanlarda tüm mortalite anlamlı olarak daha düşük (HR: 0.74, \%95 CI: $0.72-0.76, \mathrm{p}<0.0001)$ bulundu. ${ }^{[10]}$ HF-ACTION çalışmasında artan BB dozu ile orantılı olarak tüm ne- 
denli ölüm ve hastane yatışlarında azalma olduğu bu faydanın > $>50 \mathrm{mg} / \mathrm{g}$ karvedilol eşdeğeri dozlarda daha fazla artmadığı saptandı. Takipte yüksek BB dozu ve daha düşük kalp hızının tek yönlü varyans analizinde daha düşük mortalite ile ilişkili olmasına ragmen çok yönlü varyans analiz sonrası tüm nedenli ölüm ve hastane yatış1 ile sadece BB dozu ilişkii bulundu. ${ }^{[1]}$ Nitekim CIBIS II çalışmasında da bisoprololün faydası bazal kalp hızı ve takipte kalp hızındaki düşme oranından bağımsız idi. ${ }^{[12]}$ Dolayısıyla kalp yetmezliğinde tolere edilebilen yüksek BB dozuna çıkmak önemli bir hedef olmalıdır.

Kalp yetmezliğinde, PARADIGM HF ${ }^{[13]}$ çalışmas1 ile sağkalımda artış ve yaşam kalitesinde düzelme sağladığ1 gösterilen sakubitril/valsartan, 2016 ESC ve ACC/AHA Kalp Yetersizliği kılavuzları tarafından Sınıf IB endikasyonla önerilmektedir. Avrupa kılavuzu ACEİ (veya ARB), BB ve MRA kullanımına rağmen yakınmaları devam eden olgulara ACEİ yerine ARNI başlanmasını önerirken, ACC/AHA kılavuzu ACEİ veya ARB'yi tolere edebilen ve yakınmaları süren hastalarda MRA kullansın kullanmasin ARNI'ye geçilebileceğini belirtmektedir. ${ }^{[14]}$

PARADIGM-HF çalışmasında hastaların \%93'ü BB, 80\%'i diüretik, \%54'ü MRA kullanmaktaydı. Sakubitril/valsartan ile enalaprile göre KV nedenli ölüm veya $\mathrm{KY}$ nedeniyle hastane yatışında görece $\% 20$ risk azalması sağlandı. ${ }^{[13]}$ Hastaların kullanmakta olduğu BB, MRA, diuretik, dijital gibi mortalite ve KY üzerine olumlu etkileri bilinen tedavileri alıp almamasina göre bu fayda durumununun devam edip etmediğini araştıran alt analizler yapıldı. BB almayan hasta sayısı çok az olduğu için BB alan ve almayan hastalarda sakubitril/valsartanın ek faydasını kıyaslamak istatiksel olarak mümkün değildi. Bu nedenle hastalar kılavuz önerilerindeki hedef BB dozlarının (karvedilol $50 \mathrm{mg}$, bisoprolol $10 \mathrm{mg}$, metoprolol 200 $\mathrm{mg}$, nebivolol $10 \mathrm{mg}$ ) $\geq \% 50$ 'sini alan ve <\%50'sini alanlar şeklinde iki gruba ayrıldı. BB kullanan hastaların \%47'si $(n=3645)$ hedef dozun $\geq \% 50$ 'sini almaktaydı. Sakubitril/valsartanın Enalaprile görece ilave faydası hem $<\% 50$ hem de $\geq \% 50$ hedef dozunda BB alan hastalarda benzer bulundu. Enalapril verilenlere göre sakubitril/valsartan verilenlerde birincil son nokta (KV nedenli ölüm veya KY nedeniyle hastaneye yatış) hem düşük hem de yüksek BB dozu alanlarda $\% 18$ daha az idi. KV ölüm ise düşük BB dozu alanlarda $\% 15$, yüksek BB dozu alanlarda $\% 16$ daha az idi (Tablo 2) (Şekil 1). Dolayısıyla sakubitril/ valsartan'ın DEFKY de yararlı etkileri eşlik eden BB dozundan bağımsız bulundu..$^{[15]}$
Sonuç olarak, KY de sağkalım ve yaşam kalitesi üzerine faydası kanıtlanmış olan BB'ler hastaların önemli bir kısmında hedef dozda kullanılmamaktadır. Sakubitril/valsartan KY tedavisinde kullanılan BB dozundan bağımsız olarak, gerek tavsiye edilen hedef dozlarda gerekse daha düşük dozlarda $\mathrm{BB}$ alan $\mathrm{KY}$ hastalarında, ilave fayda göstermekte, ölüm ve KY nedenli hastane yatışlarını azaltmaktadır.

\section{Kaynaklar}

1. Ponikowski P, Voors AA, Anker SD, Bueno H, Cleland JGF, Coats AJS, et al. 2016 ESC Guidelines for the diagnosis and treatment of acute and chronic heart failure: The Task Force for the diagnosis and treatment of acute and chronic heart failure of the European Society of Cardiology (ESC)Developed with the special contribution of the Heart Failure Association (HFA) of the ESC. Eur Heart J 2016;37:2129-200.

2. Bhatt AS, DeVore AD, DeWald TA, Swedberg K, Mentz RJ. Achieving a Maximally Tolerated $\beta$-Blocker Dose in Heart Failure Patients: Is There Room for Improvement?. J Am Coll Cardiol 2017;69:254250 .

3. Packer M, Fowler MB, Roecker EB, Coats AJ, Katus HA, Krum $\mathrm{H}$, et al. Effect of carvedilol on the morbidity of patients with severe chronic heart failure: results of the carvedilol prospective randomized cumulative survival (COPERNICUS) study. Circulation 2002;106:2194-9.

4. Packer M, Bristow MR, Cohn JN, Colucci WS, Fowler MB, Gilbert $\mathrm{EM}$, et al. The effect of carvedilol on morbidity and mortality in patients with chronic heart failure. U.S. Carvedilol Heart Failure Study Group. N Engl J Med 1996;334:1349-55.

5. CIBIS-II Investigators and Committees. The Cardiac Insufficiency Bisoprolol Study II (CIBIS-II): a randomised trial. Lancet 1999;353:9-13.

6. MERIT-HF Study Group. Effect of metoprolol CR/XL in chronic heart failure: Metoprolol CR/XL Randomised Intervention Trial in Congestive Heart Failure (MERIT-HF). Lancet 1999;353:2001-7.

7. Fonarow GC, Abraham WT, Albert NM, Stough WG, Gheorghiade $\mathrm{M}$, Greenberg BH, et al. Dosing of beta-blocker therapy before, during, and after hospitalization for heart failure (from Organized Program to Initiate Lifesaving Treatment in Hospitalized Patients with Heart Failure). Am J Cardiol 2008;102:1524-9.

8. Düngen HD, Apostolovic S, Inkrot S, Tahirovic E, Töpper A, Mehrhof F, et al. Titration to target dose of bisoprolol vs. carvedilol in elderly patients with heart failure: the CIBIS-ELD trial. Eur J Heart Fail 2011;13:670-80.

9. Komajda M, Anker SD, Cowie MR, Filippatos GS, Mengelle B, Ponikowski $\mathrm{P}$, et al. Physicians' adherence to guideline-recommended medications in heart failure with reduced ejection fraction: data from the QUALIFY global survey. Eur J Heart Fail 2016;18:514-22.

10. Ajam T, Ajam S, Devaraj S, Sawada S, Kamalesh M. Higher betablocker dose versus lower heart rate in patients with heart failure due to systolic dysfunction. JACC 2017;69:666.

11. Fiuzat M, Wojdyla D, Kitzman D, Fleg J, Keteyian SJ, Kraus WE, et al. Relationship of beta-blocker dose with outcomes in ambulatory heart failure patients with systolic dysfunction: results from the HFACTION (Heart Failure: A Controlled Trial Investigating Outcomes of Exercise Training) trial. J Am Coll Cardiol 2012;60:208-15.

12. Lechat P, Hulot JS, Escolano S, Mallet A, Leizorovicz A, WerhlenGrandjean M, et al. Heart rate and cardiac rhythm relationships with bisoprolol benefit in chronic heart failure in CIBIS II Trial. Circulation 2001;103:1428-33. 
13. McMurray JJ, Packer M, Desai AS, Gong J, Lefkowitz M, Rizkala $\mathrm{AR}$, et al. Baseline characteristics and treatment of patients in prospective comparison of ARNI with ACEI to determine impact on global mortality and morbidity in heart failure trial (PARADIGM-HF). Eur J Heart Fail 2014;16:817-25.

14. Sarı İ, Çavuşoğlu Y, Temizhan A, Yılmaz MB, Eren M. 2016 ESC and ACC/AHA/HFSA heart failure guideline updates: Changes, simi- larities, differences, and unresolved isssues. [Article in Turkish] Turk Kardiyol Dern Ars 2016;44:625-36.

15. Okumura N, Jhund PS, Gong J, Lefkowitz MP, Rizkala AR, Rouleau JL, et al. Effects of Sacubitril/Valsartan in the PARADIGM-HF Trial (Prospective Comparison of ARNI with ACEI to Determine Impact on Global Mortality and Morbidity in Heart Failure) According to Background Therapy. Circ Heart Fail 2016;9:e003212. 